\title{
Algorithm Development for Analysis of Statistical Accuracy of the Extraction of Cartographic Features in Digital Images
}

\author{
Guilherme Pina Cardim, Erivaldo Antônio da Silva, Maurício Araújo Dias \\ FCT/UNESP - Faculty of Science and Technology - University of São Paulo State, Brazil; \\ gpcardim@gmail.com, silva.erivaldo@gmail.com, madias@fct.unesp.br
}

\begin{abstract}
Cartography uses large-scale digital images obtained by remote sensing. Such images are commonly used for the extraction and / or detection of cartographic features, which are the targets of interest in mapping. Extracting targets of interest from digital images streamlines the mapping, but the accuracy of this mapping depends on the characteristics of the features of interest present in the extracted image. Therefore, this paper proposes an automated way to calculate and display statistical values so that the extraction processes of any feature type mapping can be assessed considering its quality and quantity. With this purpose in mind, a computer program has been developed. This program applies an already established methodology to calculate statistical values concerning the results obtained by the automatic extraction process. The implemented program performs calculations in a quick and objective manner. In addition, it also generates resultant images that provide the user viewing of the errors obtained by the reported method. This paper presents the results obtained from the use of this computer program. Thus, the program developed accomplishes the proposed objectives, allowing the user to perform a consistent analysis of the automated extraction, since this evaluation is performed based on statistical calculations. Therefore, this program assists researchers and scholars of cartography to evaluate automatic extraction processes in the cartographic features of interest.
\end{abstract}

Keywords: Extraction of Cartographic Features, Evaluation Process, Digital Image Processing.

\section{INTRODUCTION}

Remote sensing images, from earth-orbiting satellites, have been widely used for several studies. These studies could be improved and automated through the use of digital image processing (DIP) [2], [17], which had its greatest impetus in the late 50's. Already in the 1960's, the U.S. launched many satellites into orbit, such as the GOES and the NIMBUS, in order to capture and send orbital images to Earth through techniques of PDI. Since then, several satellites were launched into orbit with this goal. From this point, cartographic studies that use 
these images to design computational algorithms in order to extract cartographic features in the images [19] have started. We use the term targets for cartographic features or characteristics of interest present in the digital image related to the mapping area, such as roads, rivers, and even crater pictures of planetary surfaces. Among the purposes of an extraction, we can mention the use of extracted images for mapping large areas, the cartographic updating and calculation of flooded areas, for example.

Over time, several methods of feature extraction have been developed. All aim at a methodology capable of automatically extract features from digital images from any earthorbiting satellites. Once the extraction result is obtained by the several developed methods, it is of a great importance being able to evaluate the quality obtained by this process.

In the literature, some metrics are presented to evaluate the quality achieved by an extraction method [20], however these metrics were used only for extraction of the road network. Furthermore, there are metrics which are adapted and implemented in algorithms [18], [4], but these works perform analysis without using the tolerance areas, while there is an algorithm that only uses them [3]. Another computer program found on the automation of the evaluation [14] is not in the public domain and therefore cannot be used on an ongoing basis. Besides this, the methodology and the metrics used by this program are still unclear. Thus, researchers find it difficult to qualify the developed processes of extraction. As a result, the quality of the extraction process is usually visually analyzed or by manually calculated metrics.

Therefore, this paper proposes an automated way to calculate statistical values for the extraction processes of cartographic features so that they can be assessed from qualitative and quantitative viewpoints, using or not the tolerance area. A variation of the methodology for calculating the metrics in the literature is also proposed [20].

It is justified by the necessity of using a free computer program to calculate specific statistical values related to quality analysis presented in a particular automatically performed extraction process, regardless of the type of feature mapping in this image, so that this program can be used by the user community area.

The remaining part of this paper is organized as follows. Section 2 provides the necessary definitions for the understanding of the methodology applied in this work. Section 3 presents the methodology used in the computer program to obtain statistical values. Section 4 shows the results obtained by the computer program at each step the methodology presented. The last section presents a discussion and the conclusions made in accordance with the results.

\section{THEORETICAL RECITAL}

In this section, we will provide a theoretical knowledge on the studied subject during the development of this project, such as concepts present in the literature about the evaluation process for the extraction of cartographic features and metrics related to this subject. 


\subsection{Assessment Methods of Cartographic Extraction}

Literature is scarce on how to evaluate extraction methods for cartographic features. It is commonly observed in the literature to use two methods to evaluate the results obtained during the extraction process: the overlay method and the method described by Christian Wiedmann [20], [21]. The first method is simple and performed overlapping the extracted image on the original image, thus making it possible to observe whether any feature of interest was extracted by the executed process. This process is usually carried out during the steps determination and parameters extraction tests, thus facilitating the visualization of large displacements and errors made in the implementation of the studied extraction method.

However, the superposition method does not provide a good quality metric. Thus, the method reported in the literature for determining a good quality metric for a process of extracting features of interest is the method described by Christian Wiedmann in its various publications, presented in the next section, or adaptations of these metrics [3], [4], [18].

\subsection{Quality Metrics}

An evaluation of the results obtained is essential for any automatic system [21]. Thus, it is defined that for the evaluation of an automatic method for extraction of cartographic features is always necessary a reference image [3], [4], [18], [21], which is considered as correct. It is used as basis for the statistical calculations to be performed, whose statistical results serve as a basis for the user to determine the quality of the extraction obtained with through a certain automatic method used.

In order to evaluate the extraction process, the resulting image of the extraction process is compared pixel by pixel with the reference image. The comparison is carried out with an acceptance buffer (tolerance) disposed separately on both images [20]. The method described by the author considers as coincident pixels if one is present within the acceptance buffer of the other one during the comparison.

By doing so, the author is able to count the total number of the feature points of reference, which coincides with the feature extracted (pixels matching the extracted image - Figure 1 (a)), and the extracted feature points, which coincide with the reference feature (coincident pixels in the reference image - Figure 1 (b)). As a result, the method also obtains the total points that do not coincide during the comparisons. 


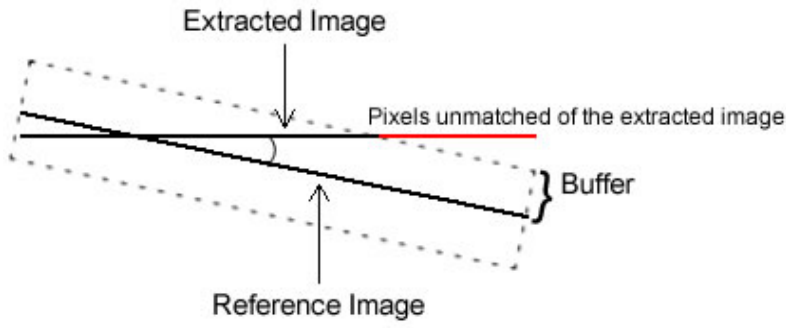

(a) Comparison of the extracted image

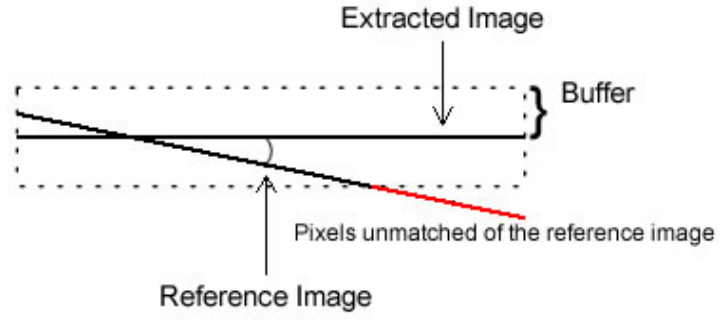

(b) Comparison of the reference image

Figure 1: Features Matching Process

Some statistical calculations are defined based on the values of the totals mentioned in the previous paragraph [20], [21]. These calculations can be used by the user as metrics to evaluate the quality obtained by the extraction of cartographic features of interest.

Referring to Figure 1, it can be noted that the comparison method shown [20] uses only the skeleton or contour of the feature of interest present in a digital image. Therefore, preprocessing is necessary in order to perform skeletonization or detecting the edges of the feature of interest. Given that the errors obtained during an automatic extraction of features are usually located on the edges of the features of interest, the section 2.3. defines an approach to edge detection.

\subsubsection{Completeness}

The first proposed quality metric is called completeness [20], [21], and the calculation is defined by the equation 1 . The completeness value represents the percentage of pixels of the reference image that has been properly extracted by the method used to obtain the extracted image. The value can vary in the interval $[0: 1]$ and the value 1 is considered optimum.

$$
\text { completeness }=\frac{\text { matched pixels of reference image comparison }}{\text { total of pixels of the reference image }}
$$

\subsubsection{Correctness}

The second quality metric is called correctness [20], [21], and its formula is given by the equation 2. This metric represents the percentage of the extracted image points that are in accordance to the reference image. The correctness can vary within the range [0:1], having the value 1 as great.

$$
\text { correctness }=\frac{\text { matched pixels of extracted image comparison }}{\text { total of pixels of the extracted image }}
$$

\subsubsection{Quality}

Once completeness and correctness metrics have been defined, a third metric called quality is presented. This metric does not include more information than what is already present in the completeness and correctness values, since the value of quality can be defined based on the 
values obtained by the first two metrics presented, as shown in equation 3. However, the value of quality is useful in cases where it is necessary to use only one value to describe the quality obtained by a particular extraction method. As in the completeness and correctness metrics, the value of quality is in the range [0:1] and 1 is expected as a great result.

$$
\text { quality }=\frac{\text { completeness }{ }^{*} \text { correctness }}{\text { complet. }- \text { complet } .{ }^{*} \text { correct. }+ \text { correct. }}
$$

\subsubsection{Redundancy}

Other metric reported in the literature is the value called redundancy [20]. Defined by the equation 4, this value represents the percentage of coincident pixels in the extraction that are redundant with each other, the ones that overlap. For this metric, the optimal value is 0 , and

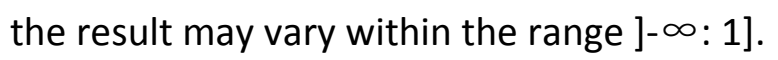

$$
\text { redundancy }=\frac{\text { matched of extraction }- \text { matched of reference }}{\text { total of extracted image }}
$$

\subsubsection{RMS}

In the literature, it is also displayed the simplified RMS metric (Root Mean Square) [20], which comprises the average difference between the overlapping pixels and the extraction reference. The result of this measure depends on the size of the buffer and usually has a normal distribution of the extracted feature on the buffer around the reference feature, allowing the use of the equation 5 to calculate the RMS. The RMS value is defined in the interval [0:buffer size], with 0 as the optimal value for this quality metric.

$$
R M S=\frac{1}{\sqrt{3}} *(\text { buffer size })
$$

\subsubsection{Simplified comparison}

Following the presented approach, a simplified comparison is accomplished without the use of buffer acceptance or tolerance feature and the total of interest without the need to find the edges of the feature, where errors tend to occur [4], [18]. This comparison is illustrated in Figure 2, originally presented in [4], in which the resulting image gets: black for non-feature points of interest; white color for pixels belonging to the feature of interest in both images; blue points that belong to the feature only in the resulting from the automated image extraction (false positives); and red for points that belong to the feature of interest only in the reference image (false negatives). 


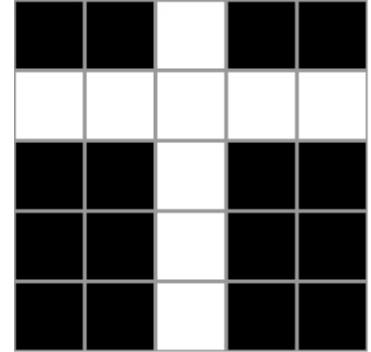

Reference

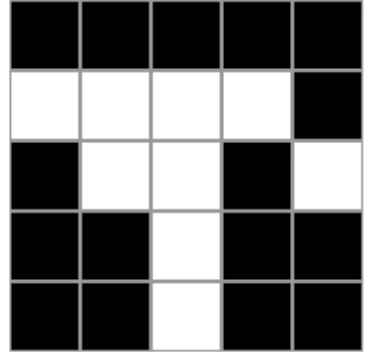

Automatic Extraction

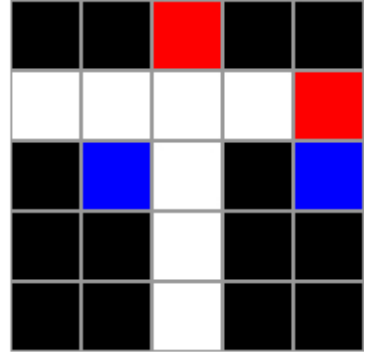

Compared Image

Figure 2: Simplified Comparison

From the resulting image, equation 6 is shown, which is called the correspondence equation (C). [4]

$$
C=\left(\frac{T W}{T W+T R+T B}\right)
$$

Where: $C$ is the correspondence value; TW is the total of white pixels; TR is the total of red pixels; and TB is the total of blue pixels.

\subsection{Edge Detection}

Edge detection is one of the most common operations for image analysis. In 1996, it was supposed to have probably more algorithms defined in the literature than any other subject in particular [15]. Although not current, the previous statement shows the importance of this subject for literature. This is because the edges are the basic characteristics of the features present in an image [17]. The edges of objects or features present in an image carry essential information about the limits of the features of interest, being of extremely importance in the characterization and analysis of images. As a result, many algorithms have been developed in order to detect the edges present in a digital image.

It is known that the edges of a target in a digital image are perceived by the great change in the digital values within the image. When there is interest in finding large changes in a onedimensional function, the best procedure is to use the theory of the second derivative, as this will be null when the magnitude value of a derivative is maximum [7]. However, this definition is not limited to a single dimension, and may be extended to two dimensional functions, such as an image. In digital images, the edge detection is defined as a local variation of the intensity of brightness.

Computationally, the gradient of an image can be calculated by performing a convolution on a particular mask on the original image. The Sobel operator is often used in this context. This fact is due to the good performance and low sensitivity to noise of the Sobel method. On the other hand, the Laplacian operator, being a second order derivate, is very sensitive to noise, for which it is rarely used in practice [16]. However, in our case, using the Laplacian operator becomes interesting, since this unwanted noise is a feature that needs to be detected by the operator. Thus, the edges detected by the use of Laplace becomes a variation of the original evaluation 
method, which uses the skeleton of the feature of interest [20]. This variation has been proposed in this work because it is considered that, in general, the errors of an extracting method are located at the edges of the feature of interest.

\subsection{Morphological Dilation}

Another concept related to PDI, whose theoretical foundation is also important for this work, is the morphologic image dilation, which expands the objects present in the image in relation to the background of it [6]. Thus, the dilation is capable of eliminating small noises on an image object. This approach is embedded in the context of the mathematical morphology, which is based on the set theory to represent and manipulate the values of a digital image [16].

Thus, it is known that for two sets defined in $R 2, A$ and $B$, the dilation of $A$ by $B$ is denoted by $A$ $\oplus B$ and defined by the equation 7 [9]. For this purpose, $B$ is assumed as a structuring element and $A$ as the set, or the image to be expanded.

$$
A \oplus B=\left\{Z \mid(\hat{B})_{Z} \cap A \neq \varnothing\right\}
$$

The expansion can be viewed as a convolution of the structuring element $B$ on the image $A$, for which the neighborhood of each feature point of interest will undergo some changes in accordance to the shape of the structuring element. Thus, the structural element has a primary role in the result generated by the expanding operator, once the size and shape of the structuring element respectively define the size and shape of the expanded area.

\section{TEST AREA OF RESEARCH}

In order to validate the methodology applied by the system, we have used a set of 15 images obtained from the database of the Faculty of Science and Technology - UNESP. In order to demonstrate this methodology, this work presents an image obtained by the panchromatic band of QuickBird satellite with a spatial resolution of $0.6 \mathrm{~m}$. This image is shown in Figure 3, which has as one feature of interest a clover from Raposo Tavares highway. 


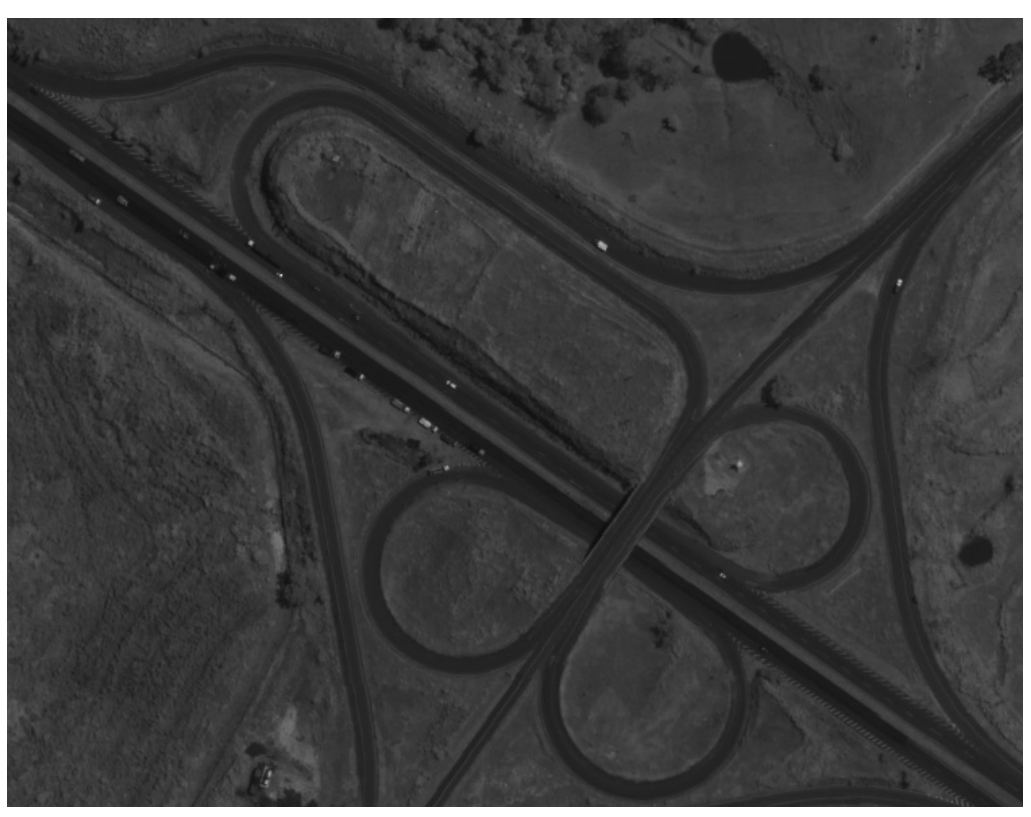

Figure 3: Original Image

Besides the original image, a reference image is required (ground truth), which will be considered as correct, or as absolute truth for statistical calculations. The reference image can be generated from measurements taken in the field. However, the reference image is usually achieved manually by the use of an image editor software, selecting all points belonging to the cartographic feature of interest in the original image [4]. Therefore, as an example in this work, the reference image was manually obtained and is shown in Figure 4.

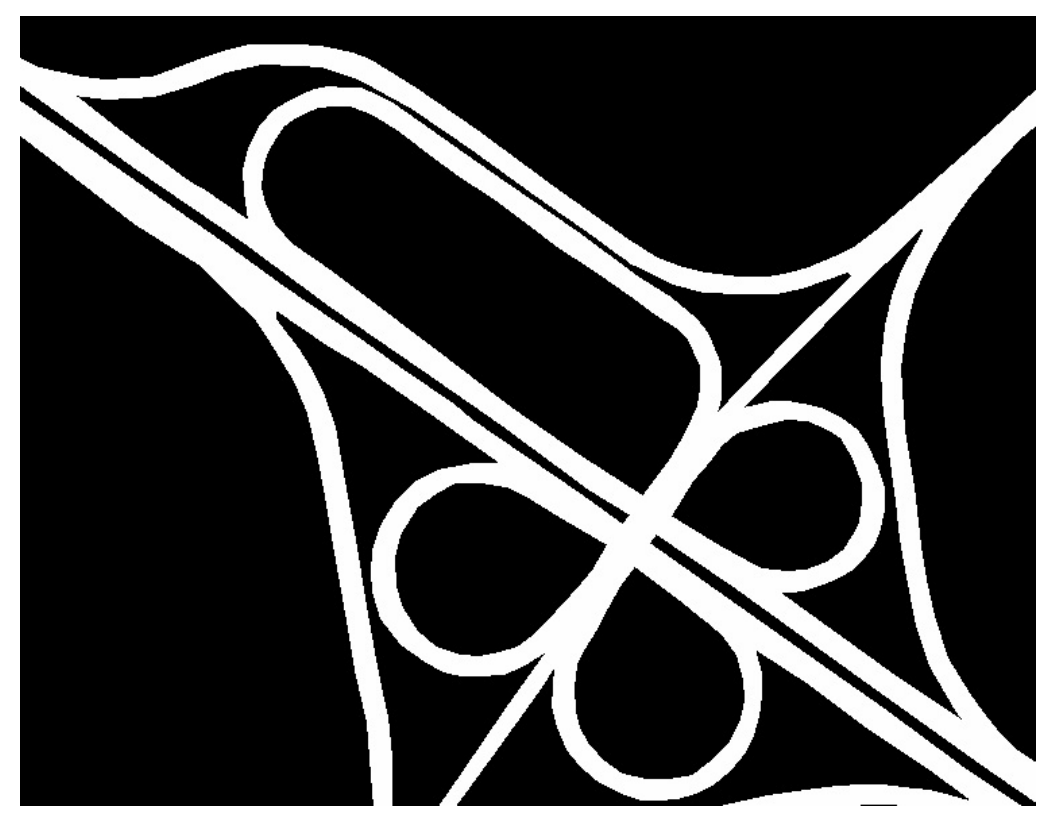

Figure 4: Reference Image 
The computational proposed program also requires the resulting image from the process of automatic extraction so that it can be statistically analyzed. Thus, the image obtained by an automatic extraction method for the example is shown in Figure 5.

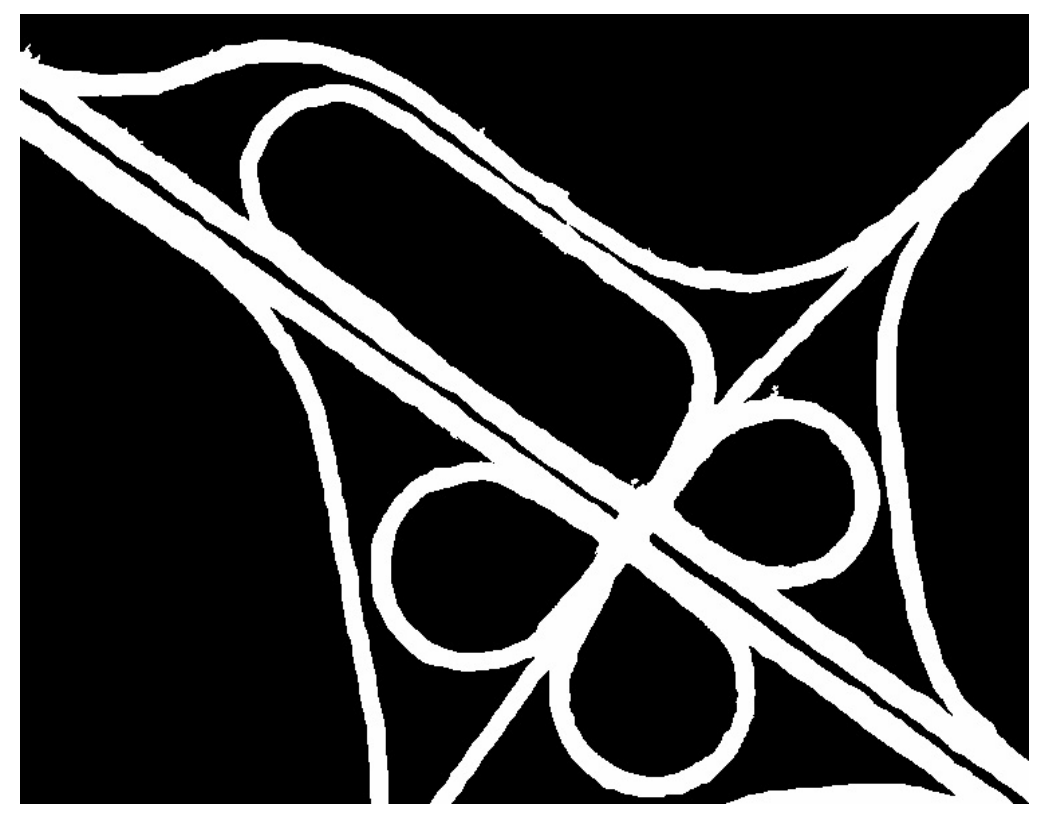

Figure 5: Automatic Extracted image

\section{METHODOLOGY}

The methodology applied in the computer program is developed based on results from the use of the metrics presented in the section 2.2. For this purpose, it is necessary to use a reference image, the resulting image from the automated extraction process, the original image used to obtain the reference and the extracted images.

Having the three images and knowing that the errors of an extraction process concentrate on the edges of the feature of interest, the first step of the evaluation methodology applied is to obtain the edges of the feature of interest present in the reference and extracted images. For this purpose, the Laplacian operator is applied over the image to detect any change in brightness values, since this filter behaves like a second order derivative being sensitive to any change in the values of the digital image. Thus, the Laplacian filter has shown as appropriate in order to detect the edges of the feature of interest, since the reference and extracted images are binary, which makes it easy to find modifications of brightness values of the digital image.

After obtaining the images containing the edges of the feature of interest, both in the reference as in the automatically extracted images, the second stage of the methodology is to check the coordinates or the position of each point belonging to the edges. These coordinates are used to fetch the gray levels in the original image, enabling the performance of traditional statistical calculations such as mean, mode, median, standard deviation and minimum and maximum values. 
Having calculated the first statistical values, the third step of the evaluation method is to make a comparison between the obtained edges for the reference and the extracted images. The comparison is carried out pixel by pixel with no tolerance among the images, generating a resultant image, as exemplified by Figure 2 . The resulting image of this comparison enables the visualization of coincident points and errors found during this process. Performed the comparison, the computer program gets the number of matching points and points that were classified as false positives (pixels blue) or false negatives (pixels red). Thus, it becomes possible to perform the calculations of the correspondence metric (Equation 6), of completeness (Equation 1) and the correctness (Equation 2), where the tolerance is regarded as zero for the calculation of the last two metrics.

However, as described in the literature [20], [21], the comparison can be performed using a tolerance, defined as a buffer acceptance for positioning the edge pixels of the feature of interest. Accordingly, the fourth step of the methodology consists of generating the tolerance range for the reference and extracted images by implementing morphological dilation operation on the images containing the edges of the feature of interest and reference images extracted. In order to perform the morphological operator, the user needs to define the structural element to be used and hence the shape and size of the tolerance generated by the program around the feature of interest.

The fifth step of the methodology applied by the program is to compare the image containing the edges of the feature of interest from the reference image with the image containing the dilated edges of the feature of interest of the extracted image. During the comparison, the program calculates the points that coincide or not, calling them reference coincident or not coincident points. Similarly, a comparison is also made of the image containing the extracted image edges of the feature of interest with the image containing the enlarged edges of the feature of interest from the reference image. In this comparison, the match points are called extraction coincidents and, consequently, the ones which do not match are called extraction non-coincident. In both comparisons, the program generates a resulting image containing errors or mismatched points found during each comparison.

Having calculated the reference and the extraction coincident or not coincident points, on the sixth stage of the methodology the program calculates the Equations 1, 2, 3, 4 and 5 in order to get the statistical values that influence the quality obtained by the automatic extraction of cartographic features. All calculated values enable the user to perform a statistical evaluation of the quality resulted by the automatic extraction performed to obtain the extracted image.

\section{PRESENTATION AND ANALYSIS OF THE RESULTS}

To demonstrate the methodology used by the program, this article presents one example, as mentioned above, for which the original, the reference and the extracted images are displayed respectively on Figures 3, 4 and 5. 
As a first result of the methodology, we calculate the statistical values of mean, median, mode, standard deviation, minimum and maximum values of the brightness values of the original image according with the edges positions of the reference and extracted images. The values obtained for the sample are shown in Table 1.

Table 1: Traditional Statistics

\begin{tabular}{|l|l|l||}
\hline & Reference & Extracted \\
\hline \hline Mean & 54.437 & 53.427 \\
\hline Median & 54 & 54 \\
\hline Mode & 54 & 51 \\
\hline Minimum & 18 & 28 \\
\hline Maximum & 158 & 151 \\
\hline Standard Deviation & 8.93232 & 9.06013 \\
\hline
\end{tabular}

Continuing the methodology, the program performed the statistical calculations concerning the comparison without image tolerance containing the edges of the feature of interest of the reference and extracted images. Therefore, the obtained values for the example are presented in Table 2 and Figure 6 shows the result image.

Table 2: Comparison Without Buffer of Tolerance

\begin{tabular}{||l||l||}
\hline & Comparison without tolerance \\
\hline \hline Total Matched & 2349 \\
\hline Total of False Positives & 6623 \\
\hline Total of False Negatives & 6499 \\
\hline Completeness & 0.265484 \\
\hline Correctness & 0.261815 \\
\hline Correspondence & 0.151832 \\
\hline
\end{tabular}




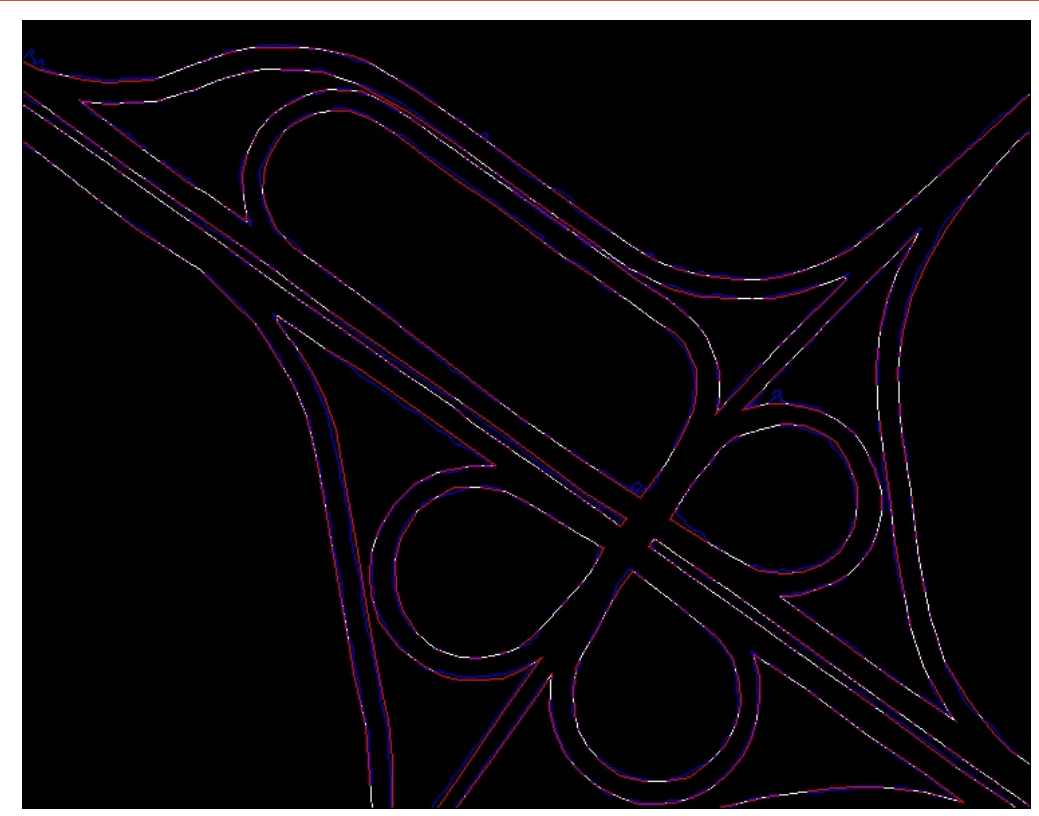

Figure 6: Result of Simplified Comparison

After, in order to generate the images of the edges with tolerances by morphological dilation, as described in the fourth step of the method, a structure element with dimensions $3 \times 3$ cross type was used, generating o point of tolerance at neighborhood 4.

As part of the fifth stage of the methodology, a comparison between the images with the edges of the reference image with the dilated image of the extracted image edges was performed. Figure 7 shows the image resulting from this comparison. In this image, the red points are those who have not corresponded in the comparison made between the images, being considered as false negatives.

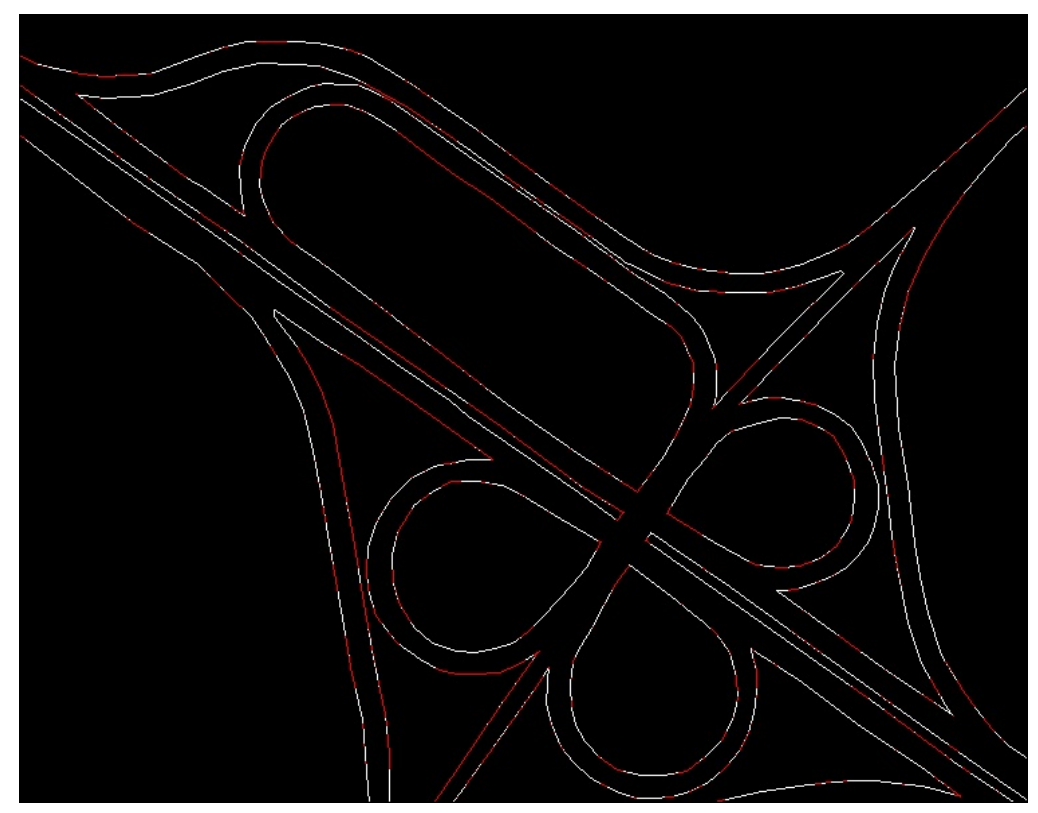

Figure 7: Comparison of Reference Over Extracted Image 
Finishing the fifth step, the reverse comparison was also performed. A comparison between the image of the automatically extracted image edges with the image of the performed dilation on the edges of the reference image was made. The red points, in this case, are considered false positives because they have not corresponded in this comparison. The image is shown in Figure 8.

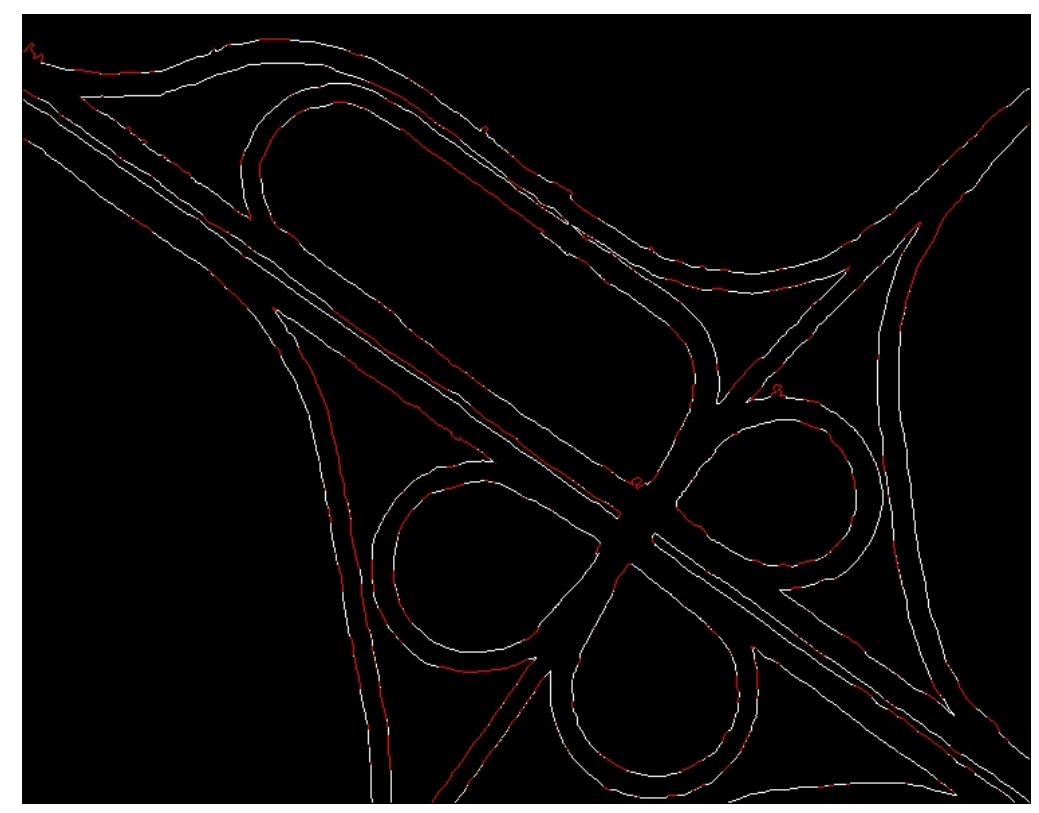

Figure 8: Comparison of Extracted Over Reference Image

Having performed these comparisons, the computer program calculated the related metrics compared with the tolerance area. The results calculated by the program are presented in Table 3. It is noteworthy that the structuring element used in the example was the type cross with dimensions $3 \times 3$.

Table 3: Comparison With Buffer of Tolerance

\begin{tabular}{|l||l||}
\hline & Comparison with tolerance \\
\hline \hline Matched in the Extracted Image & 5985 \\
\hline Unmatched in the Extracted Image & 2987 \\
\hline Matched in the Reference Image & 5982 \\
\hline Unmatched in the Reference Image & 2866 \\
\hline Completeness & 0.67608 \\
\hline Correctness & 0.66707 \\
\hline Quality & 0.50557 \\
\hline Redundancy & 0.0005 \\
\hline RMS & 1.1547 \\
\hline \hline
\end{tabular}




\section{CONCLUSIONS}

With the purpose of obtaining an automated way to calculate and display statistical values related to the results obtained by an automatic method of extracting cartographic features, a free computer program capable of performing the statistical calculations from the original image, from a reference image and from a resulting image from the extraction process to be evaluated has been developed.

Facing the need of automation of statistical calculations regarding the quality obtained by the extraction process, the software was able to remedy the limitations imposed by the researched studies [3], [4], [18], [20]. This is observed by the metrics automating related to comparisons with or without using tolerance through the implementation of a computer system. The importance of using the proposed qualitative and quantitative evaluations of automatic methods for extraction of cartographic features program was evidenced by the results obtained during the implementation of the program for the example shown. The program, for example, has noted that traditional statistics obtained for the reference images and extracted (Table 1) and the values of tolerance without comparison performed on these images (Table 2) did not allow a more accurate assessment of the quality achieved by the cartographic features automatic process extraction in the example shown. This is due to the high ratio between the obtained data for the reference and the extracted images in traditional statistical calculations, and the low values obtained for the comparison of images without tolerance. Thus, the statistical values related to the comparison performed by utilizing the tolerance range were to be analyzed.

Concerning the obtained values for the example, when using a tolerance of only one point, the statistical obtained values were not satisfactory for the automatic extraction of cartographic features, since quality metrics have shown that only $50 \%$ of the points agreed between the reference image and the image resulting from the automatic extraction process. However, we should remember that the tolerance used was just one pixel that is equivalent to $0.6 \mathrm{~m}$ at the surface. Increasing the tolerance, the values should get better. This way, the values help the user in the evaluation, who becomes responsible for the final analysis of the evaluated extraction method.

In conclusion, the developed program has demonstrated its effectiveness by providing statistical data related to the quality of the obtained extract, facilitating the analysis of the user on the performed extraction method. The results obtained with the developed computer program confirm the importance of evaluating methods of automatic feature extraction.

The computational program contributes to the literature for the following reasons: it is able to calculate statistical values related to analysis of the quality of a procedure for automatic extraction, statistical calculations are carried out efficiently and quickly (the maximum waiting time for results was less than one (1) second, and the time depends on the size of the images and the comparison tolerances); it performs few comparisons during processing, since such 
Guilherme Pina Cardim, Erivaldo Antônio da Silva and Maurício Araújo Dias; Algorithm Development for Analysis of Statistical Accuracy of the Extraction of Cartographic Features in Digital Images; Transactions on Machine Learning and Artificial Intelligence, Volume 2 No 2 Apr (2014); pp : 32-47

comparisons are performed on binary images; resulting images are generated in each matching process, which facilitates the visualization of the errors obtained in the positioning of these on the image; it allows a traditional statistical analysis on the original image; and moreover, the developed system is not restricted and may be operated by any user.

Therefore, it is concluded that the program developed assists the user to perform qualitative and quantitative assessments quickly and consistently in relation to the automatic extraction process executed, since these ratings are based on the studied statistical calculations and on the images resulting from the comparisons performed by the program.

All tests performed during this work were done on remote sensing images containing cartographic features of interest, because this is a common type of images used in Cartography. However, we intend to conduct further studies of the application of the same computer program for aerial photographs and even tests using others edge detectors.

\section{REFERENCES}

[1]. Banon, G. J. F. Course at "X Congresso Nacional de Matemática Aplicada e Computacional", Gramado, 1987.

[2]. Baxes, G. A. “Digital Image Processing: principles and applications", John Willey, New York, 1994.

[3]. Cardim, G. P.; Silva, E. A. Development of na algorithm to analyze cartographic features extraction methods, in "35 International Symposium on Remote Sensing of Environment”, ISPRS, Maryland, 2013.

[4]. Cardim, G. P.; Silva, E. A. Análise da qualidade de processos automáticos de extração de feições cartográficas, Omnia Exatas, 2011, 4(2): p. 7-18.

[5]. Crósta, A. P. "Processamento Digital de Imagens de Sensoriamento Remoto", IG/UNICAMP, Campinas, 1999.

[6]. Davies, E. “Computer and Machine Vision: Theory, Algorithms, Practicalities", Academic Press, 2012.

[7]. Forsyth, D. A; Ponce, J. “Computer Vision - a modern approach”, Prentice Hall, 2003.

[8]. Gomes, J.; Velho, L. “Computação Gráfica: imagem”, IMPA, Rio de Janeiro, 1994.

[9]. Gonzalez, R. C.; Woods, R. E. "Processamento de Imagens - técnicas digitais", Pearson Prentice Hall, New Jersey, 2008.

[10]. Ishikawa, A. S. “Detecção de Rodovias em Imagens Digitais de Alta Resolução com o Uso da Teoria da Morfologia Matemática", Master Project, FCT/UNESP, Presidente Prudente, SP, 2008.

[11]. Lampinen, J.; Laaksonen, J.; Oja, E. "Remote Sensing and Image Interpratation", John Wiley \& Sons, 2008. 
[12]. Lampinen, J.; Laaksonen, J.; Oja, E. “Pattern Recognition”, Image Processing and Pattern Recognition, Academic Press, 1998.

[13]. Muller, D. N.; Daronco, E. L. “Operações Aritméticas em Imagens”. Universidade Federal do Rio Grande do Sul, Porto Alegre, 2000.

[14]. Nóbrega, R. A. A. "Deteç̧ão de Malha Viária na Periferia Urbana de São Paulo Utilizando Imagens Orbitais de Alta Resolução Espacial e Classificação Orientada a Objetos", PhD Thesis. Escola Politécnica, USP, São Paulo, 2007.

[15]. Parker, J. R. “Algorithms for Image Processing and Computer Vision”, John Wiley \& Sons, 1996.

[16]. Pedrini, H.; Schwartz, W. R. "Análise de Imagens Digitais: princípios, algoritmos e aplicações", Thomson Learning, São Paulo, 2008.

[17]. Pitas, I. "Digital Image Processing Algorithms and Applications", John Wiley \& Sons, 2000.

[18]. Silva, E. A.; Cardim, G. P. Applying Digital Image Processing to Evaluate an Extraction Method of Cartographic Features in Digital Images, Journal of Earth Science and Engineering, 2012, 2(4): p. 241246.

[19]. Silva, E. A.; Leonardi, F. Calculation of the Area of Power Plant Using Mathematical Morphology, Journal of Communication and Computer, 2012, 9(2): p. 221-225.

[20]. Wiedemann, C. External Evaluation of Road Networks, ISPRS Archives, 2003, XXXIV: p. 93-98.

[21]. Wiedemann, C.; Heipke, C; Mayer, H.; Hinz, S. Automatic Extraction and Evaluation of Road Networks from Moms-2p Imagery, International Archives of Photogrammetry and Remote Sensing, 1998: p. 95100. 